\title{
DERECHO AL TRABAJO, AUTOMATIZACIÓN LABORAL Y DERECHOS DE AFECTACIÓN POR EL USO DE TECNOLOGÍA*
}

\author{
RIGHT TO WORK, LABOR AUTOMATION AND RIGHTS \\ OF AFFECTATION BY TECHNOLOGY'S USE
}

\author{
DROIT AU TRAVAIL, AUTOMATISATION DU TRAVAIL ET DROITS \\ D'AFFECTION POUR L'UTILISATION DE LA TECHNOLOGIE
}

\author{
Jesús AguiLERA DuRÁN**
}

\begin{abstract}
RESUMEN: El uso de la tecnología trae aparejada la utilización de robots y la inteligencia artificial en diversos ámbitos de la producción de bienes y la prestación de servicios, lo que va generalizando la automatización laboral y el desvanecimiento de los derechos laborales de las personas, por lo que se hace necesario un replanteamiento de las expectativas profesionales y legales para el futuro. Se tienen como objetivos los siguientes: abordar la realidad y normativa del derecho al trabajo que está dirigida a regir las relaciones laborales sólo entre personas; exponer algunos datos sobre la automatización laboral y el replanteamiento que se debe hacer para enfrentar la construcción de una nueva forma de educar para un futuro no lejano; abordar la interacción del ser humano, particularmente la del trabajador, con la tecnología, y, de forma general, de ésta con las personas. Asimismo, reflexionar sobre cuándo las personas resientan alguna afectación en sus derechos por el uso de tecnología sin que la legislación contemple disposiciones para protegerlos y garantizarlos. Para alcanzar estos objetivos se plantea un relevamiento de información en fuentes bibliográficas y digitales que permitan reforzar la postura del autor sobre el contexto actual y futuro del derecho al trabajo, la diseminación de la automatización laboral y, como corolario, lo que se considera de más
\end{abstract}

* Recibido el 26 de febrero de 2019 y aceptado para su publicación el 25 de marzo de 2019.

* Profesor de Derechos Humanos y Derecho Constitucional en la Facultad de Derecho y Ciencias Sociales de la Universidad Autónoma del Estado de Morelos.

\footnotetext{
IS Revista Latinoamericana de Derecho Social

Núm. 29, julio-diciembre de 2018, pp. 3-23
} 
trascendencia: la visualización de los derechos de afectación por el uso de tecnología como derechos humanos.

Palabras clave: derechos laborales, automatización laboral, robots, derechos de afectación.

ABSTRACT: The use of technology brings with it the use of robots and artificial intelligence in various areas of the production of goods and the provision of services, which is generalized labor automation and the fading of labor rights of people, so that It is necessary to rethink professional and legal expectations for the future. The following objectives are aimed at: addressing the reality and regulations of the right to work that is aimed at governing labor relations only between people; expose some data on labor automation and the rethinking that must be done to face the construction of a new way of educating for a future not far away; to approach the interaction of the human being, particularly the worker with the technology, and in a general way, of this with the people. Also, reflect on when people resent any impact on their rights for the use of technology without the legislation includes provisions to protect and guarantee them. To achieve these objectives, a survey of information in bibliographic and digital sources is proposed to reinforce the author's position on the current and future context of the right to work, the dissemination of labor automation and, as a corollary, what is considered to be transcendence, the visualization of rights of affectation due to the use of technology as human rights.

Keywords: labor rights, labor automation, robots, rights of affectation.

RÉSUMÉ: L'utilisation de la technologie entraîne l'utilisation de robots et de l'intelligence artificielle dans divers domaines de la production de biens et de la fourniture de services, à savoir l'automatisation généralisée du travail et la disparition progressive des droits des personnes en matière de travail. ce qui devient nécessaire est de repenser les attentes professionnelles et juridiques pour l'avenir. Les objectifs suivants visent à: faire face à la réalité et aux réglementations du droit au travail visant à régir les relations de travail uniquement entre personnes; exposer quelques données sur l'automatisation du travail et les réflexions à faire pour faire face à la construction d'une nouvelle manière d'éduquer pour un avenir proche; aborder l'interaction de l'être humain, en particulier du travailleur, avec la technologie et, d'une manière générale, avec les gens. En outre, réfléchissez au moment où les gens ressentent un quelconque impact sur leurs droits à l'utilisation de la technologie sans que 
la législation comprenne des dispositions visant à les protéger et à les garantir. Pour atteindre ces objectifs, une enquête sur l'information figurant dans des sources bibliographiques et numériques est proposée pour renforcer la position de l'auteur sur le contexte actuel et futur du droit au travail, de la diffusion de l'automatisation du travail et, en corollaire, de ce qui est considéré comme un droit. transcendance, la visualisation des droits d'affectation résultant de l'utilisation de la technologie en tant que droits de l'homme.

Mots-clés: droits du travail, automatisation du travail, robots, droits d'affectation.

SuMARIO: I. Nota introductoria. II. El derecho al trabajo en el contexto juridico mexicano. III. El derecho al trabajo en el ámbito internacional. IV. Automatización de las profesiones para el trabajo. V. Interacción de humanos con robots en el campo laboral VI. ¿Y si hablamos de una responsabilidad por uso de la tecnología? VII. Visualización de los derechos por afectación por del uso de tecnología como derechos humanos. VIII. Conclusiones. IX. Fuentes de información.

\section{NOTA INTRODUCTORIA}

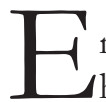
$\mathrm{n}$ un contexto internacional donde el reconocimiento de los derechos humanos se va dando a cuentagotas, lo que hace cada vez más complicado poder acceder a su ejercicio pleno, se presentan situaciones de conflicto en el ámbito nacional para poder mantener su protección y garantía, ya que casi por lo general se tiene que acudir al ámbito jurisdiccional para poder exigirlos.

Sirva lo anterior para resaltar que el Estado no ha garantizado a cabalidad el derecho de acceso a la justicia, que en ocasiones es negado o no está al alcance de las personas por falta de recursos económicos para pagar un proceso judicial, que va a ser largo, costoso, y que de ninguna forma garantiza alcanzar justicia a sus pretensiones. ${ }^{1}$

1 Cabrera Dircio, Julio y Aguilera Durán, Jesús, "La justicia alternativa, el derecho colaborativo y sus perspectivas en México", Cuestiones Constitucionales. Revista Mexicana de Derecho Constitucional, núm. 40, enero-junio de 2019, p. 247, disponible en: http:// dx.doi.org/10.22201/ iij.24484881e.2019.40. 
En ese contexto, se va perdiendo la eficacia de los derechos laborales, ya que los resultados de esa histórica lucha por el aseguramiento de condiciones laborales que provean a los trabajadores de una seguridad y estabilidad laborales se antoja cada vez más difícil de mantener, porque se van desvaneciendo los derechos adquiridos o su vigencia en la normativa laboral.

Además, los ámbitos laborales se ven influenciados cada vez más por los avances de la tecnología.

La evolución de las telecomunicaciones y de la informática ha sido tan vertiginosa que ha llegado incluso a modificar el marco normativo previsto para las relaciones laborales. Es por ello que la protección de los derechos fundamentales se haya alzado como plano prioritario, siendo objeto de una regulación exhaustiva y de su correspondiente interpretación por el colectivo comunitario. ${ }^{2}$

El uso de la tecnología trae aparejada la utilización de robots y la inteligencia artificial en diversos ámbitos de la producción de bienes y la prestación de servicios, lo que va generalizando la automatización laboral, por lo que se hace necesario un replanteamiento de las expectativas profesionales para el futuro, porque a los dueños del capital les representa más ganancias invertir en robots que mejorar las condiciones de trabajo para sus empleados.

"No nos cabe la menor duda que la robótica y la inteligencia artificial traerán consigo eficiencia y ahorro en todos los sectores (producción y comercio, transporte, asistencia sanitaria, educación y agricultura, etc.) pero también pueden causar que aquéllos asuman gran parte del trabajo que ahora realizamos los humanos". ${ }^{3}$

Con esa automatización laboral, también se presenta la interacción robothumanos, de la cual se van a derivar diversas situaciones en las que el trabajador puede resentir alguna afectación en sus derechos o en su persona por el uso de la tecnología en los diversos sectores productivos de la sociedad, incluida la inminente readaptación de los seres humanos a nuevas condiciones laborales.

Dicha afectación, que puede ser de tipo físico, normativo, psicológico o laboral, en lo que respecta a pérdidas o disminución en los derechos de los

2 Monereo Pérez, José Luis y López Insua, Belén del Mar, “BIB 2014\122”, Revista Doctrinal Aranzadi Social, núm. 11, Cizur Menor, 2014, p. 28.

3 Sierra Benítez, Esperanza Macarena, "El trabajo digital y la robótica en la Unión Europea", en Cerejeira Namora, Nuno et al., Digital Work and Personal Data Protection, Cambridge Scholars Publishing, 2018, p. 68. 
trabajadores, también puede resentirse en otros aspectos de la vida cotidiana de las personas cuando entran en contacto con el uso de la tecnología.

Esto plantea la necesidad de realizar una evaluación de las consecuencias éticas y morales de las nuevas tecnologías, porque los robots deben respetar los lógicos derechos fundamentales de las personas y los derechos sociales protegidos. Para ello, se deben prever las medidas técnicas que garanticen el respeto en la propia fase de diseño (protección de la intimidad desde el momento inicial), así como establecer unas consideraciones sobre seguridad (información y regulación de la responsabilidad derivada del uso, como quien es el propietario, quien tiene la potestad para disponer de ellos y quien puede modificar su implantación). ${ }^{4}$

En esa tesitura, este artículo tiene como objetivos los siguientes: abordar la realidad y normativa del derecho al trabajo, que está dirigido a regir las relaciones laborales sólo entre personas; exponer algunos datos sobre la automatización laboral y el replanteamiento que se debe hacer para enfrentar la construcción de una nueva forma de educar, para un futuro no lejano; abordar la interacción del ser humano, particularmente del trabajador con la tecnología, y de forma general, de ésta con las personas. Asimismo, como corolario, lo que se considera de más trascendencia: hacer visibles los derechos de afectación por el uso de tecnología como derechos humanos.

Para alcanzar estos objetivos se plantea un relevamiento de información en fuentes bibliográficas y digitales, que permitan reforzar la postura del autor sobre el contexto actual del derecho al trabajo, la diseminación de la automatización laboral y la visualización de los derechos de afectación por el uso de tecnología.

\section{EL DERECHO AL TRABAJO EN EL CONTEXTO JURÍDICO MEXICANO}

Los derechos sociales, los laborales entre ellos, se plasmaron por primera vez en la Constitución Política de los Estados Unidos Mexicanos (CPEUM) de 1917, la que se denominó "la primera Constitución social en el mundo".

En Latinoamérica varias Constituciones siguieron los pasos del constitucionalista mexicano e incorporaron a su texto los derechos sociales, lo que

4 Ibidem, pp. 80 y 81. 
significó entender al ser humano como un individuo inserto en un contexto social, con necesidad de una protección más amplia frente a las vicisitudes de la vida que debe afrontar en condiciones propias de la dignidad del ser humano. ${ }^{5}$

Con esa visión, las personas pertenecientes a sectores vulnerables de la sociedad, en aras de proteger su dignidad ante las desigualdades sociales, reciben un reconocimiento del Estado, que les va a permitir tener una vida decente. Uno de estos sectores lo representan los trabajadores, que ven cristalizada esa visión a través de los derechos laborales.

El derecho al trabajo encuentra su sustento en los artículos 1o., como matriz de los derechos humanos, y en el 123, que es propio de las relaciones laborales; asimismo, en la Ley Federal del Trabajo y en la Ley Federal de los Trabajadores al Servicio del Estado.

Sin embargo, con los avances de la tecnología se percibe un desvanecimiento de los derechos laborales; por ejemplo, la estabilidad en el trabajo o la seguridad social, que se han visto seriamente disminuidas con las nuevas formas de contratación laboral.

Se deberá prever la formación y capacitación de nuevos puestos laborales, así como nuevos riesgos o enfermedades profesionales derivados de los mismos puestos a los cuales se tendrá que hacer frente. Aunado a lo anterior, el reto se encuentra en encontrar el equilibrio entre desarrollo tecnológico y el respeto a los derechos de los trabajadores; así como implementar la protección que necesita el trabajo de la era digital. ${ }^{6}$

Un factor que provoca otra afectación seria en los derechos del trabajador es la ocasionada por el poderío económico de las empresas trasnacionales, que se allegan de abogados para cabildear reformas legislativas que favorecen a sus intereses o esquivar a las leyes laborales.

En lo que respecta a la CPEUM, se resalta que si bien en el artículo 1o. se reconocen los derechos humanos, los derechos laborales entre ellos, también se imponen obligaciones a las autoridades de promover, respetar, proteger y

5 Kurczyn Villalobos, Patricia, "Seguridad social”, en Fix-Zamudio, Héctor y Valadés, Diego (coords), Instituciones sociales en el constitucionalismo contemporáneo, 2a. ed., México, UNAMColegio Nacional, 2011, p. 202.

6 Mendizábal Bermúdez, Gabriela y López Pérez, Emmanuel, “¿Nuevo modelo de seguridad social en el contexto de la industria 4.0?”, Revista Internacional y Comparada de Relaciones Laborales y Derecho del Empleo, vol. 6, núm. 1, enero-marzo de 2018, p. 315. 
garantizar los derechos humanos, de conformidad con los principios de universalidad, interdependencia, indivisibilidad y progresividad.

Con relación al principio de progresividad, se entiende que la obligación estatal consiste en ir adaptando a la realidad el respectivo avance en los derechos o la inclusión de nuevos derechos en la legislación interna. Además, el artículo 1o. contiene el principio pro persona y el principio de interpretación conforme, lo que amplía el ámbito de protección sobre los derechos humanos.

En ese orden de ideas, la CPEUM en el artículo 123 prescribe que "Toda persona tiene derecho al trabajo digno y socialmente útil; al efecto, se promoverán la creación de empleos y la organización social de trabajo, conforme a la ley". Con esta disposición se reconoce el derecho al trabajo, y también se estipulan los deberes del Estado en este ámbito; pero nada se habla de responsabilidad con relación al uso de robots o la inteligencia artificial en el ámbito laboral.

El artículo 123 es una de las conquistas de la clase obrera en la Revolución mexicana, y, por lo mismo, se establece como una norma de mínimos para proteger al trabajador en contra del patrón. Esta misma tendencia se observa en su ley reglamentaria (la Ley Federal de Trabajo), y en las normas laborales de Latinoamérica. ${ }^{7}$

En la fracción XIV de este precepto se menciona que "los empresarios serán responsables de los accidentes del trabajo y de las enfermedades profesionales de los trabajadores, sufridas con motivo o en ejercicio de la profesión o trabajo que ejecuten"; sin embargo, en la descripción de ambos conceptos no existe ninguna alusión de responsabilidad que surja de la interacción trabajador-robot.

En el apartado A se regulan las relaciones laborales "entre los obreros, jornaleros, empleados domésticos, artesanos y de una manera general, todo contrato de trabajo"; en el apartado B, las relaciones laborales "entre los Poderes de la Unión y sus trabajadores". Este artículo es el fundamento de la Ley Federal del Trabajo (LFT) y de la Ley Federal de los Trabajadores al Servicio del Estado (LFTSE), las cuales van a detallar todo lo relacionado con el derecho laboral.

7 Mendizábal Bermúdez, Gabriela, "Estudio de derecho comparado de la inspección del trabajo en Latinoamérica", Revista Latinoamericana de Derecho Social, núm. 28, enero-junio de 2019, p. 162, disponible en: http:/ / dx.doi.org/10.22201/iij.24487899e.2019.28.13147. 
La LFT rige las relaciones de trabajo comprendidas en el apartado A del artículo 123. En otras palabras, las relaciones laborales del sector privado. De tal modo que detalla todas las disposiciones de dicho apartado.

$\mathrm{El}$ artículo 6o. dispone que "Las Leyes respectivas y los tratados celebrados y aprobados en los términos del artículo 133 de la Constitución serán aplicables a las relaciones de trabajo en todo lo que beneficien al trabajador, a partir de la fecha de la vigencia".

En cuanto a la responsabilidad, por ejemplo, es de poner atención a lo que dispone el artículo 489, que prescribe:

No libera al patrón de responsabilidad:

I. Que el trabajador explícita o implícitamente hubiese asumido los riesgos de trabajo;

II. Que el accidente ocurra por torpeza o negligencia del trabajador; y

III. Que el accidente sea causado por imprudencia o negligencia de algún compañero de trabajo o de una tercera persona.

En la fracción III se considera pertinente que debería agregarse "o de alguna máquina o robot".

La LFTSE, también conocida como la ley burocrática, es reglamentaria del apartado B del artículo 123 constitucional, y como ya se mencionó rige las relaciones laborales entre los trabajadores del Estado y los poderes e instituciones enunciadas en su artículo primero.

Así, la jornada laboral se estipula como de ocho horas durante el trabajo diurno, de siete horas para el nocturno y la mixta de siete horas y media como máximo. No se hace mención alguna a la interacción que se produce entre los humanos y los robots en el trabajo.

El artículo 46 dispone:

Ningún trabajador podrá ser cesado sino por justa causa. En consecuencia, el nombramiento o designación de los trabajadores sólo dejará de surtir efectos sin responsabilidad para los titulares de las dependencias por las siguientes causas:

V. Por resolución del Tribunal Federal de Conciliación y Arbitraje, en los casos siguientes:

f) Por comprometer con su imprudencia, descuido o negligencia la seguridad del taller, oficina o dependencia donde preste sus servicios o de las personas que allí se encuentren. 
Por virtud de este inciso f), el trabajador sí puede ser sancionado hasta con el cese de sus funciones, pero nada se aborda sobre la posibilidad de que resienta una afectación por descuido o negligencia de un robot o máquina.

La automatización y la digitalización perfilan, además, otras consecuencias de dimensión social, entre otras: un aumento temporal del desempleo o la consolidación de un nivel de desempleo estructural significativo que genere necesidades de protección social más amplias, dado el número de personas afectadas por el proceso disruptivo. ${ }^{8}$

Del análisis breve sobre la legislación laboral más importante en nuestro país se aprecia que es necesaria una adecuación a una realidad cada vez más cotidiana sobre el uso de la tecnología en el ámbito laboral, puesto que el uso de robots y de la inteligencia artificial se vuelve cada vez más frecuente en nuestro entorno, sin que se actualice manifestación legal alguna sobre dicho fenómeno.

\section{EL DERECHO AL TRABAJO EN EL ÁMBITO INTERNACIONAL}

En lo que respecta al ámbito universal, se puede decir que el fundamento de los derechos laborales se encuentra en el Pacto Internacional de Derechos Económicos, Sociales y Culturales y en la Organización Internacional del Trabajo; en la región americana, en el Protocolo Adicional a la Convención Americana de Derechos Humanos en materia de Derechos Económicos, Sociales y Culturales.

En dichos instrumentos jurídicos internacionales, al igual que en el ámbito nacional, no se visualiza una inclusión normativa sobre las relaciones donde interactúan los humanos con los robots, ni en el campo laboral ni en términos generales.

El Pacto Internacional de Derechos Económicos, Sociales y Culturales, en su artículo 6.1, reconoce el derecho a trabajar, el cual se complementa con el reconocimiento al derecho de toda persona al goce de condiciones de trabajo equitativas y satisfactorias del artículo 7o.; con la obligación de los

8 Bonet Pérez, Jordi, "Disrupción tecnológica y trabajo, ¿disrupción también en el ámbito de las relaciones laborales?”, Revista Mientras Tanto.e, núm. 169, junio de 2018, p. 4, disponible en: http:// www.mientrastanto.org/boletin-169/notas/disrupcion-tecnologica-y-trabajo-disrupcion-tambienen-el-ambito-de-las-relaciones (fecha de consulta: 11 de febrero de 2019). 
Estados, de garantizar la libertad sindical del artículo 8o. y el reconocimiento del derecho de toda persona a la seguridad social y al seguro social plasmado en el artículo 9o.

Sin embargo, en la era digital, la automatización de diversas actividades laborales, el trabajo a distancia, varios de estos derechos no se protegen ni se garantizan por los Estados, lo que conlleva el desvanecimiento de los derechos laborales para los trabajadores que entran en contacto con la tecnología.

Por lo que toca a la Organización Internacional del Trabajo, como máximo órgano especializado en la materia laboral, se considera pertinente tomar en cuenta sus predicciones:

Los avances tecnológicos - la inteligencia artificial, la automatización y la robótica — crearán nuevos puestos de trabajo, pero quienes van a perder sus trabajos en esta transición podrían ser los menos preparados para aprovechar las nuevas oportunidades. Las competencias de hoy no se ajustarán a los trabajos de mañana y las nuevas competencias adquiridas pueden quedar desfasadas rápidamente. ${ }^{9}$

Esta recomendación resulta muy atinada, porque más que preocuparse por las incertidumbres del futuro, hay que ocuparse en prepararse hoy en día para asumir el reto de estar profesional, técnica y mentalmente capacitado para incursionar en áreas laborales, que tal vez en nuestro pasado reciente no visualizábamos como parte de nuestra ocupación laboral o de nuestras vidas.

Por esa razón, la OIT propone un programa centrado en las personas para el futuro del trabajo y recomienda centrar los esfuerzos en aumentar la inversión en las capacidades de las personas, aumentar la inversión en las instituciones del trabajo e incrementar la inversión en trabajo decente y sostenible. ${ }^{10}$

Con ese rediseño de las políticas laborales se hará necesario un ajuste en la regulación laboral, que brinde certeza jurídica y seguridad a los trabajadores.

El Protocolo Adicional a la Convención Americana sobre Derechos Humanos en Materia de Derechos Económicos, Sociales y Culturales contempla los derechos laborales en el artículo 6o., las condiciones de trabajo justas, equitativas y satisfactorias en el artículo 7o.; los derechos sindicales en el artículo 8o. y el derecho a la seguridad social en el artículo 9o.

9 Comisión Mundial sobre el Futuro del Trabajo, Trabajar para un futuro más prometedor, Oficina Internacional del Trabajo, Ginebra, OIT, 2019, p. 10.

10 Ibidem, pp. 11-13. 
Tampoco en este convenio se visualiza alguna disposición que extienda estos derechos fuera de la formalidad en el trabajo, ya que no se contemplan los derechos de aquellos empleados que trabajando en casa no tienen protección sobre la seguridad social, o de aquellos trabajandores que no pueden acceder a un seguro de desempleo al verse desplazados de sus fuentes de trabajo por la automatización laboral.

\section{AutOMATIZACIÓN DE LAS PROFESIONES PARA EL TRABAJO}

El uso de la tecnología en las fuentes de trabajo es cada vez más frecuente, lo que está provocando la automatización de las profesiones; unas presentan mayores afectaciones que otras, principalmente, aquellas de baja cualificación; aunque también las especializadas están en riesgo; pero no cabe duda alguna sobre el impacto real de la injerencia del uso de la robótica y la inteligencia artificial en las expectativas profesionales para el trabajo.

"De acuerdo a nuestro análisis de más de 2,000 actividades laborales en 800 profesiones, cerca de la mitad de las actividades por las cuales se pagan salarios equivalentes a $\$ 15$ billones en la economía mundial tienen el potencial de ser automatizadas si se adoptan tecnologías probadas". ${ }^{11}$ En ese orden de ideas, se considera que las actividades más proclives a la automatización son las físicas predecibles, el procesamiento de datos y la compilación de datos.

Esto sin duda representa un gran reto para la sociedad en su conjunto, puesto que si no se corrige la forma de educar o trabajar, las afectaciones en el sector laboral se verán tanto para los jóvenes como para los adultos, ya que se requiere estudiar con responsabilidad, capacitarse constantemente y pensar que el trabajar no sólo representa la ilusión de tener un puesto, sino reflexionar si se está cualificado para siquiera pensar en obtenerlo.

"Aunque menos del 5 por ciento de todas las profesiones pueden ser automatizadas en su totalidad usando tecnologías probadas, cerca del 60 por ciento de todas las profesiones están integradas por actividades automatizables y que representan por lo menos el 30\% de su total". ${ }^{12}$ En este 5\% se incluyen

11 Mckinzey Global Institute, "Un futuro que funciona: automatización, empleo y productividad", resumen ejecutivo 2017, disponible en: https://mww.mckinsey.com/ / medial mckinsey/featured\%20 insights / digital\%20 disruption / harnessing\%20automation \%20for $\% 20$ a\%20 future\%20that\%20works/ a-future-that-works-executive-summary-spanish-mgi-march-24-2017.ashx' (fecha de consulta: 14 de febrero de 2019).

12 Idem. 
profesiones relacionadas con el sector de hospedaje y prestación de servicios, almacén, agencias de viajes, contabilidad, administración, preparación de impuestos y manufactura.

"Los grandes volúmenes de datos y aprendizaje automático harán que sea posible automatizar muchas tareas que eran difíciles de automatizar en el pasado". ${ }^{13}$

Por lo que se hace necesario prepararse, tanto académicamente como desarrollar habilidades, destrezas y capacidades que permitan ser más competitivos en cualquier área laboral que se tiene pensado incursionar.

Por el contrario, empleos altamente cualificados que implican las capacidades de resolución de situaciones, la intuición y la creatividad, y tareas que se realizan «en persona» y que precisan de ciertas destrezas y habilidades de comunicación social flexible para una mejor prestación de servicios (atención, trato, etc...), son más difíciles de convertir en rutina. ${ }^{14}$

Por lo tanto, la automatización va a ser influida por el tipo de actividad que se realiza.

En resumen, las tareas rutinarias se definen como tareas que siguen reglas explícitas que pueden ser realizadas por las máquinas, mientras que las tareas no rutinarias no son suficientemente comprendidas como para ser especificadas en el código de computadora. Cada una de estas categorías de tareas puede ser, a su vez, de naturaleza manual o cognitiva — por ejemplo, si se relacionan con el trabajo físico o el trabajo del conocimiento-. ${ }^{15}$

En cuanto a la profesión jurídica, aún persiste la duda si la labor que realiza un operador jurídico, cuya función principal es la toma decisiones, puede ser reemplazada en su totalidad por un robot, puesto que la experiencia, la preparación académica, el raciocinio y su visión personal son factores que influyen en su desempeño profesional.

13 Mercader Uguina, Jesús R., "El impacto de la robótica y el futuro del trabajo", Revista de la Facultad de Derecho de México, t. LXVII, núm. 269, septiembre-diciembre de 2017, p. 158, disponible en: http://dx.doi.org/10.22201/fder.24488933e.2017.269.62438 (fecha de consulta: 19 de febrero de 2019)

14 Idem.

15 Benedikt Frey, Carl y Osborne, Michael, "The Future of Employment: How Susceptible are Jobs to Compurezitation?”, Oxford Martin Programme on Technology and Employment, septiembre de 2013, pp. 16 y 17, disponible en: https://mwn.oxfordmartin.ox.ac.uk/downloads/ academic/The_Future_of_Employment.pdf(fecha de consulta: 21 de febrero de 2019). 
Muchos criterios de decisión son subjetivos del juzgador, y por ello, muy difícilmente codificables para ser procesados por un sistema informatizado (la apreciación sobre la verosimilitud de un testigo o la suficiencia de una prueba). Además, parece que difícilmente una máquina podría ser capaz de responder a una cuestión no previamente contemplada por el ordenamiento (el problema de la anomia o la falta de precedentes para un caso). ${ }^{16}$

Aunado a que los abogados van a litigar casos que aunque parecieran similares, cada uno goza de ciertas particularidades, vicisitudes o complejidad, puesto que cada persona tendrá sus propias reacciones, que a veces son impredecibles; cada abogado asume su estrategia, y los resultados van a diferir, dependiendo de cómo se desarrolle el proceso.

¿Pero podrán estos sistemas constituirse en herramientas de apoyo a los profesionales jurídicos? Muy probablemente sí. La posibilidad de contar con sistemas capaces de analizar a gran velocidad enormes volúmenes de documentación, para extraer de ellos pautas o mejores opciones de actuación ante supuestos concretos, parece posible y de gran utilidad para los usuarios. ${ }^{17}$

Con esto, los robots pueden representar una ayuda significativa, mas no reemplazar la capacidad humana, dotada de raciocinio natural y del discernimiento, que permite tomar decisiones, tomando en cuenta todos y cada uno de los factores que inciden en el análisis, síntesis y resolución de un caso. Además, estará ausente la valoración emocional, que en no pocas veces es lo que complica la resolución de un caso concreto.

Por lo antes expuesto, la educación y las políticas públicas deben enfocarse a la consolidación de profesiones que son poco susceptibles de automatización o la creación de nuevas profesiones relacionadas con la utilización de la tecnología en el campo laboral, tomando en consideración las que los expertos consideran más relevantes.

Destacan los perfiles tecnológicos o digitales cualificados (analistas de Big Data, perfiles orientados a resolver problemas complejos, especialistas en redes sociales o híbridos entre diseño y tecnología); perfiles relacionados con el

16 Fernández Hernández, Carlos y Boulat, Pierre, "Inteligencia artificial y derecho: Problemas y perspectivas", Noticias Jurídicas, 2015, disponible en: http:// noticias.juridicas.com/ conocimiento/articulos-doctrinales/9441-inteligencia-artificial-y-derecho-problemas-y-perspectivas/ ( fecha de consulta: 20 de febrero de 2019).

17 Idem. 
trato con personas (psicólogos, coaches y facilitadores en empresas o gestores de nuevas formas de trabajo); y perfiles de ventas y marketing (especialistas cualificados en desarrollo de negocio y vendedores técnicos)..$^{18}$

Sólo de esta forma se estarán formando profesionistas cualificados para un futuro laboral influenciado por el uso de la tecnología en la producción de bienes y la prestación de servicios, específicamente de los robots y la inteligencia artificial.

\section{INTERACCIÓN DE HUMANOS CON ROBOTS \\ EN EL CAMPO LABORAL}

Como se ha expuesto en líneas anteriores, con la automatización de diversas profesiones se provocan dos efectos: el primero, sobre la deshumanización de la mano de obra, y el segundo sobre el desvanecimiento de los derechos laborales de los trabajadores, que al verse avasallados por la tecnología no alcanzan a comprender que o se renuevan en sus capacidades, conocimientos, destrezas y habilidades, o se quedarán sin empleo en un futuro cercano.

Ahora bien, se considera muy buena idea el hacer uso de la robótica y de la inteligencia artificial en el trabajo, pero con ese uso, necesariamente se presenta una interacción cotidiana de los robots con los humanos, lo que puede ocasionar que se presente una afectación en sus derechos o incidentes, donde, seguramente, el humano se verá en inferioridad de condiciones en relación con los robots.

"El eje central de la reflexión y, por tanto, la proyección jurídica es o ha de ser el mantenimiento de la identidad humana y su dignidad (y en torno a ellas sus derechos irrenunciables) junto a la mejora del ser humano a través de la tecnología y el respeto de los derechos humanos". ${ }^{19}$

Dicho en otras palabras, en esa interacción, los robots, además de desplazar a los trabajadores de tareas que eran propias de sus actividades diarias,

18 Repositorio institucional de la Fundación Universitaria San Pablo CEU, Informe ADDECO sobre el futuro del trabajo en España, 2016, p. 138, disponible en: https:/ / wmw.ceu.es/ joblab/documentacion/informeAdecco.pdf (fecha de consulta: 10 febrero de 2019).

19 Sánchez-Urán, Ma. Yolanda, "El impacto de la robótica en especial la robótica inclusiva en el trabajo: aspectos jurídico-laborales y fiscales", estudio realizado en el marco de los proyectos CertificaRSE, DER 2015-65374-R (MINECO-FEDER); e INBOTS CSA, Inclusive Robotics for a better Society, Programa H2020-ICT-2017-1; proyecto 780073, disponible en: bttp:/ / inbots.eu/wp-content/ uploads/2018/08/publications/robotica-derecho-del-trabajo-derecho-fiscalfinal-mayo2018.pdf. 
puede conducir a situaciones en las que los humanos resientan alguna afectación física o psicológica, puesto que la impotencia de verse rebasados en conocimientos les puede producir estrés laboral.

Por otro lado, los trabajadores en contacto con los robots pueden sufrir accidentes o enfermedades que les produzca alguna afectación en su integridad, ya sea física o intelectual.

Con la diseminación del uso de los robots será necesario replantear el papel que deben asumir los trabajadores para poder ser competitivos y poder acceder a un empleo. Será necesario echar mano de todos los recursos a su alcance. Me parece que en este punto, los trabajadores de edades avanzadas sufrirán más las consecuencias de haber nacido en una época anterior a la revolución tecnológica.

Se considera indiscutible la idea de que en el campo laboral se prevé que los empresarios inviertan cada vez más en la robótica como una forma de agilizar la productividad, pero también como una forma de tener menos obligaciones contractuales con los trabajadores. En ese caso la empresa tiene como objetivo obtener los mayores beneficios posibles.

La era digital requiere que los ciudadanos la tomen con el debido respeto, lo que los va a llevar a prepararse más y mejor, no solamente estudiando una carrera, sino complementándola con formación en informática o desarrollando habilidades para ser más competitivos. Al Estado le corresponde extender la protección de la ley a la realidad laboral.

"En resumen, no parece factible detener la digitalización, pero la política tiene muchas opciones para influir y dirigir el proceso, invirtiendo en educación, infraestructura e I + D [investigación más desarrollo], y adaptando la legislación laboral, las políticas fiscales y de seguridad social a la digitalización". ${ }^{20}$

\section{VI. ¿Y SI HABLAMOS DE UNA RESPONSABILIDAD POR USO DE LA TECNOLOGÍA?}

En la interacción humano-humano, parece cosa fácil deducir quién tendría la culpa o responsabilidad en caso de presentarse incidentes relacionados con el trabajo, sería A o sería B. Pero en la interacción de humanos con robots,

20 Dachs, Bernhard, The Impact of New Technologies on the Labour Market And The Social Economy, European Parliament, 2018, p. 53. 
cuando el humano fuera $\mathrm{C}$ y el robot $\mathrm{D}$, ¿cómo se podría deducir quién tuvo la culpa si cada uno tenía encomendadas tareas específicas? Pues eso habrá de depender de los hechos, pero de una forma u otra el trabajador podría resultar afectado.

Si D por alguna razón desvía el curso de su actividad automatizada y le inflige una herida a C, éste resentiría la afectación no sólo en su persona, sino también en su estabilidad emocional. No obstante, D no sentiría ninguna afectación, porque carece de esa parte emocional que sólo los humanos tenemos.

El inciso AB del apartado denominado "Responsabilidad" dice que

Considerando que, cuanto más autónomos sean los robots, más difícil será considerarlos simples instrumentos en manos de otros agentes (como el fabricante, el operador, el propietario, el usuario, etc.); que esta circunstancia, a su vez, suscita la cuestión de si la normativa general sobre responsabilidad es suficiente o si se requieren normas y principios específicos que aporten claridad sobre la responsabilidad jurídica de los distintos agentes y su responsabilidad por los actos y omisiones de los robots cuya causa no pueda atribuirse a un agente humano concreto, y de si los actos u omisiones de los robots que han causado daños podrían haberse evitado. ${ }^{21}$

Desde luego que es de tomar en cuenta lo antes expuesto, ya que una falla en la actividad programada puede ocasionar daños de carácter irreversible, y aunque se podría decir que el propietario del robot o el empresario es el responsable directo, puede haber más personas involucradas y resultar que no sea posible la determinación de la responsabilidad.

Considerando que, en el supuesto de que un robot pueda tomar decisiones autónomas, las normas tradicionales no bastarán para generar responsabilidad jurídica por los daños ocasionados por el robot, ya que no permitirán determinar la parte que ha de hacerse cargo de la indemnización, ni exigir a dicha parte que repare el daño ocasionado. ${ }^{22}$

En otras palabras, es imperante la necesidad de que las normas vayan evolucionando con la realidad; no se puede solucionar problemas nuevos con

21 Parlamento Europeo, Informe con recomendaciones destinadas a la Comisión sobre Normas de Derecho Civil sobre Robótica (2015/2103(INL)), Propuesta de resolución del Parlamento Europeo. Responsabilidad, inciso AB.

22 Ibidem, inciso AF. 
disposiciones normativas que datan del siglo pasado. Tampoco es pertinente que se visualice una regulación que incluya sólo uno o varios aspectos de la problemática.

Por ejemplo, en la región europea, la Directiva 85/374/CEE solamente aborda la responsabilidad por los daños causados por productos defectuosos. Lo que no es aceptable, se impone que la adecuación sea integral para que tome en cuenta la responsabilidad desde el punto de vista patrimonial, de derechos laborales o fiscales

Por lo que "deberá estudiarse la posibilidad de someter a impuesto el trabajo ejecutado por robots o exigir un gravamen por el uso y mantenimiento de cada robot, a fin de mantener la cohesión social y la prosperidad". ${ }^{23}$

Con el fin de lograr el propósito planteado en el párrafo anterior, la inclusión de una responsabilidad por uso de la tecnología, en particular, debe velar por preservar la integridad física e intelectual de las personas y, en lo general, de forma más amplia, por sus derechos, humanos.

Y el régimen de responsabilidad civil que se adopte habrá de ser preciso para regular los daños ocasionados por robots dotados de autonomía, puesto que existe una gran complejidad en el posible reparto de culpas entre los diferentes agentes involucrados, sumándose además la complejidad resultante de la posible acción originadora de daño, que se puede denominar "la culpa del robot", de difícil imputación, y que con más razón exigirá un régimen de responsabilidad objetiva y/o solidaria frente al tercero perjudicado. ${ }^{24}$

\section{VISUALIZACIÓN DE LOS DERECHOS POR AFECTACIÓN DEL USO DE TECNOLOGÍA COMO DERECHOS HUMANOS}

De actualizarse las predicciones de expertos en el área de la tecnología, los robots tenderán a ir absorbiendo un porcentaje significativo en la mayoría de las actividades, lo que puede desembocar en pérdida de empleos o un reacomodo de ellos, pero también va a ocasionar afectaciones a aquellos analfabetos digitales, trabajadores en interacción con robots, personas que usan dispositivos electrónicos, contratantes de bienes o servicios y a las personas

23 Ibidem, Introducción, inciso K.

24 Castro Calvo, Manuel, "La acción autónoma del robot”, Legal Today, 2018, disponible en: http:// wmw.legaltoday.com/opinion/articulos-de-opinion/la-accion-autonoma-del-robot (fecha de consulta: 25 de febrero de 2019). 
desplazadas de sus empleos. Asimismo, es inevitable el incremento de la interacción cotidiana entre humanos y robots.

Las modalidades de interacción son distintas entre sí — daños, relación contractual, injerto o implante en el cuerpo- y evocan otras ramas del sistema jurídico. El robot diseñado para la asistencia en el hogar o en una clínica hospitalaria entra en íntimo contacto con la persona que lo utiliza (con frecuencia con un paciente $\mathrm{o}$, en general, una persona en condiciones de fragilidad, debido a su edad, a una enfermedad o a una incapacidad), con sus familiares y con los operadores de ese ambiente, suscitando de forma inevitable la ocasión para la ocurrencia de accidentes, lesiones o perjuicios. ${ }^{25}$

De tal modo que esos accidentes, lesiones o perjuicios van a producir una afectación en los derechos de las personas, la cual puede ser física, pero extendiéndose a daños psicológicos, económicos o sociales, los que hoy en día resulta muy complejo hacerlos exigibles, puesto que la legislación se ha visto rebasada por la realidad.

Lo anterior invita a la reflexión sobre si es tiempo de ir poniendo en la agenda el replanteamiento de los derechos humanos, porque no se trata de establecer si son derechos individuales, colectivos o de solidaridad, sino de visualizar aquellos derechos de las personas que van a ser afectados por el uso de la tecnología, los que se podrían empezar a conceptualizar como los derechos de una persona que resiente una disminución o menoscabo en su integridad física o emocional, sus bienes o derechos, producto del uso de la tecnología, sea por interacción laboral, contacto o a distancia en caso de haber solicitado una prestación o servicio digital.

\section{CONCLUSIONES}

El derecho al trabajo, de poco en poco se va quedando sin dientes para morder un hueso duro de roer: el uso de la tecnología en la producción; se requiere que la regulación laboral vaya adaptándose a las nuevas tendencias que imponen el uso de la tecnología como medio para eficientizar recursos humanos o económicos; no es posible que las leyes laborales cada vez sean menos

25 Palmerini, Erika, "Robótica y derecho: sugerencias, confluencias, evoluciones en el marco de una investigación europea", Revista de Derecho Privado, núm. 32, enero-junio de 2017, p. 58, disponible en: https://revistas.uexternado.edu.co/index.php/derpri/article/view/5021/6009 (fecha de consulta: 16 de febrero de 2019). 
protectoras de los trabajadores y más condescendientes con los empresarios, mientras que el Estado parece aplicar la filosofía de laissez faire, laissez.passer.

De igual forma, se hace necesario que ante el avance imparable de la automatización en las profesiones, la educación en nivel medio superior y superior se visualice en prospectiva, no sólo en el sistema tecnológico, sino también en el universitario, para que se inserten materias sobre informática, programación y sistemas computacionales en los planes de estudio, se inserten nuevas carreras o se modifiquen las actuales y se prepare a los estudiantes y futuros trabajadores en un contexto de automatización laboral, uso de la inteligencia artificial y capacitación constante.

Con el uso de la tecnología en el ámbito laboral resulta indispensable que se hable de una responsabilidad por el uso de la tecnología en el sector productivo y de servicios, para que aquellas personas que resientan una afectación en su persona o en sus derechos puedan ser beneficiadas por disposiciones legales que les restituyan en sus derechos o las indemnicen por cualquier afectación.

En ese orden de ideas, es menester visualizar los derechos de afectación por el uso de tecnología como derechos humanos, que salvaguarden no sólo la integridad física de las personas, sino también la psicológica, económica y social, por lo que el reconocimiento de estos derechos podrá brindar una seguridad jurídica a los individuos en una sociedad cada vez más ávida de justicia ante la avasallante invasión de la tecnología en la cotidianeidad de sus vidas.

\section{FUENTES DE INFORMACIÓN}

BONET PÉREZ, Jordi, “Disrupción tecnológica y trabajo, ¿disrupción también en el ámbito de las relaciones laborales?”, Mientras Tanto.e, núm. 169, junio de 2018, disponible en: http:/ / wnw.mientrastanto.org/ boletin-169/ notas / disrupcion-tecnologica-y-trabajo-disrupcion-tambien-en-el-ambito- de-las-relaciones (fecha de consulta: 11 de febrero de 2019).

BenedikT, Carl y Osborne, Michael, "The Future of Employment: How Susceptible are Jobs to Compurezitation?”, Oxford, Martin Programme on Technology and Employment, disponible en: https: / www.oxfordmartin. ox.ac.uk/downloads/academic/The_Future_of_Employment.pdf (fecha de consulta: 21 de febrero de 2019).

Cabrera Dircio, Julio y Aguilera Durán, Jesús, "La justicia alternativa, el derecho colaborativo y sus perspectivas en México", Cuestiones Cons- 
titucionales, núm. 40, enero-junio de 2019, disponible en: http://dx.doi. org/10.22201/iij.24484881e.2019.40.

Castro Calvo, Manuel, “La acción autónoma del Robot”, Legal Today, 2018, disponible en: http:/ / www.legaltoday.com/ opinion/articulos-de-opinion/la-accionautonoma-del-robot (fecha de consulta: 25 de febrero de 2019).

COMisión Mundial sobre EL Futuro Del Trabajo, Trabajar para un futuro más prometedor, Ginebra, Oficina Internacional del Trabajo, OIT, 2019.

DACHS, Bernhard, The Impact of New Technologies on the Labour Market and the Social Economy, European Parliament, 2018.

FERnÁndeZ HernándeZ, Carlos y Boulat, Pierre, "Inteligencia artificial y derecho: problemas y perspectivas", Noticias Jurídicas, 2015, disponible en: http:/ / noticias.juridicas.com/ conocimiento/articulos-doctrinales/9441-inteligenciaartificial-y-derecho-problemas-y-perspectivas/ (fecha de consulta: 20 de febrero de 2019).

KurcZyn Villalobos, Patricia, "Seguridad social”, en FiX-ZAMudio, Héctor y VALADÉS, Diego (coords.), Instituciones sociales en el constitucionalismo contemporáneo, 2a. ed., México, UNAM-El Colegio Nacional, 2011.

MCKINZEY GLOBAL INSTITUTE, Un futuro que funciona: automatización, empleo y productividad, disponible en: https:// www.mckinsey.com/ / media/mckinsey/featured\%20insights/digital\%20disruption/ harnessing\%20automation\%20 for $\% 20$ a\%20future\%20that\%20works/ a-future-that-works-executive-summaryspanish-mgi-march-24-2017.ashx' (fecha de consulta: 14 de febrero de 2019).

MENDizÁBAl BERMúdeZ, Gabriela, "Estudio de derecho comparado de la inspección del trabajo en Latinoamérica", Revista Latinoamericana de Derecho Social, núm. 28, enero-junio de 2019, disponible en: http://dx.doi. org/10.22201/iij.24487899e.2019.28.13147.

MendizÁBAl BermúdeZ, Gabriela y LóPez PÉReZ, Emmanuel, “¿Nuevo modelo de seguridad social en el contexto de la industria 4.0?", Revista Internacional y Comparada de Relaciones Laborales y Derecho del Empleo, vol. 6, núm. 1, enero-marzo de 2018.

Mercader Uguina, Jesús R., "El impacto de la robótica y el futuro del trabajo", Revista de la Facultad de Derecho de México, t. LXVII, núm. 269, septiembre-diciembre de 2017, disponible en: http://dx.doi.org/10.22201/ fder.24488933e.2017.269.62438 (fecha de consulta: 19 de febrero de 2019).

Monereo Pérez, José Luis y LóPez InsuA, Belén del Mar, Revista Doctrinal Aranzadi Social, núm. 11, Cizur Menor, 2014. 
PALMERINI, Erika, "Robótica y derecho: sugerencias, confluencias, evoluciones en el marco de una investigación europea", Revista de Derecho Privado, núm. 32, enero-junio de 2017, disponible en: https:// revistas.uexternado. edu.co/index.php/derpri/article/view/5021/6009 (fecha de consulta 16 de febrero 2019).

PARLAMENTO EUROPEO, Informe con recomendaciones destinadas a la Comisión sobre normas de derecho civil sobre robótica (2015/2103(INL)), Propuesta de resolución del Parlamento Europeo. Responsabilidad, disponible en: http:// wnw.europarl.europa.eu/doceo/document/A-8-2017-0005_ES.html?redirect.

REPOSITORIO INSTITUCIONAL DE LA FUNDACIÓN UNIVERSITARIA SAN PABLO CEU, Informe ADDECO sobre el futuro del trabajo en España, 2016, disponible en: https:/ / www.ceu.es/joblab/documentacion/informeAdecco.pdf (fecha de consulta: 10 de febrero de 2019).

SÁNCHEZ-URÁN, Ma. Yolanda, "El impacto de la robótica, en especial la robótica inclusiva en el trabajo: aspectos jurídico-laborales y fiscales", estudio realizado en el Marco de los Proyectos CertificaRSE, DER 201565374-R (MINECO-FEDER); e INBOTS CSA, Inclusive Robotics for a better Society, Programa H2020-ICT-2017-1; Proyecto 780073, disponible en: http://inbots.eu/wp-content/uploads/2018/08/publications/roboticaderecho-del-trabajo-derecho-fiscal-final-mayo2018.pdf.

SIERRA BENITEZ, Esperanza Macarena, "El trabajo digital y la robótica en la Unión Europea", en CerejeIra NAMORA, Nuno et al., Digital Work and Personal Data Protection, Cambridge Scholars Publishing, 2018. 